\title{
IMPACTOS DAS MUDANÇAS NO PROCESSO DE TRABALHO DOS PROFISSIONAIS DE SAÚDE: O QUE DIZ A LITERATURA ${ }^{1}$
}

\author{
Emanusa Soele Santana Lessa Pereira \\ Especialista em Saúde do Trabalhador pelo \\ Instituto de Saúde Coletiva-UFBA, \\ Fisioterapeuta da Secretaria Estadual de \\ Saúde da Bahia-SESAB, Salvador, Bahia, \\ Brasil. \\ Ana Cláudia Conceição da Silva \\ Mestre em Saúde Coletiva, Professora \\ Assistente, Coordenadora e Pesquisadora \\ do Núcleo de Estudo e Pesquisa em Saúde \\ do Trabalhador e Desigualdades em Saúde \\ -NEST, Universidade Estadual do Sudoeste \\ da Bahia-UESB, Jequié, Bahia.
}

Endereço para correspondência: anaclaudiacs@gmail.com

\begin{abstract}
RESUMO
Foi realizada uma revisão de literatura com o objetivo de avaliar os processos de trabalho em saúde e sua relação com os processos de saúde e doença destes profissionais. Foram usados os descritores: "processo de trabalho em saúde", "reestruturação produtiva", "reestruturação hospitalar" e "condições de trabalho em saúde", nas bases de dados Medline, Lilacs e Scielo. Foram incluídos 19 artigos de 1999 a 2009, que abordavam temas sobre os impactos das mudanças no processo de trabalho e na saúde dos trabalhadores da atenção à saúde. Os resultados indicaram que há um processo de adoecimento do profissional de saúde, devido às conseqüências trazidas pela reestruturação. Foram apontadas como causas: duplas jornadas de trabalho, baixa remuneração, alta sobrecarga de trabalho, falta de autonomia, subordinação ao modelo médico-hegemônico atual e pressões dos chefes e familiares dos usuários, refletindo na qualidade de vida do profissional e no atendimento. Sugere-se um maior diálogo e apoio dos gestores, para serem conhecidas as lacunas e sugestões sobre o processo de trabalho, e investimento nas relações interpessoais na equipe multidisciplinar, a fim de que cada um tenha maior autonomia no trabalho. Também é necessário investir no espaço relacional trabalhador-usuário, com ações de acolhimento e humanização do atendimento.
\end{abstract}

Palavras-chave: Reestruturação produtiva; Processo de trabalho em saúde; Processo saúde-doença em saúde.

\section{IMPACTS OF CHANGES IN WORK PROCESS OF HEALTH PROFESSIONALS: WHAT THE LITERATURE SAYS}

\begin{abstract}
It was performed a literature review with the aim of evaluating the processes in health work and its relation to the processes of health and disease, of these professionals. It was used the descriptors: " process of work in health", "productive restructuring", "hospital restructuring" and "working conditions in health" in Medline, Lilacs and SciELO. It was included 19 articles from 1999 to 2009, which addressed issues on the impacts of changes in the labor process and in health of workers in health care. The results indicated that there is a disease process of the health care professional due to the consequences brought about by the restructuring. Have been identified as causes: double working hours, low pay, high workload, lack of autonomy, subordination to the medical-hegemonic current model and the pressures of chiefs and family of users, reflecting on quality of life of the professional and in care. It is suggested a greater dialogue and support from managers, to be known the gaps and suggestions on the work process, and investment in interpersonal relationships in the multidisciplinary team,
\end{abstract}

\footnotetext{
${ }^{1}$ Trabalho de Conclusão do Curso de Especialização em Saúde do Trabalhador-CEST, do Instituto de Saúde Coletiva da Universidade Federal da Bahia, realizado em parceria com o Centro Estadual de Referência em Saúde do Trabalhador.
} 
so that each one has greater autonomy at work. It is also necessary to invest in worker-user relational space, with shares host and humanization of care.

Keywords: Productive restructuring; Process of work in health; Process of health-disease in health.

\section{IMPACTOS DE LOS CAMBIOS EN EL PROCESO DE TRABAJO DE LOS PROFESIONALES DE LA SALUD: LO QUE DICE LA LITERATURA}

\section{RESUMEN}

Se realizó una revisión bibliográfica con el objetivo de evaluar los procesos de trabajo en salud y su relación con los procesos de salud y enfermedad de estos profesionales. Se utilizaron los descriptores: "proceso de trabajo en salud", "reestructuración productiva", "reestructuración del hospital" y "condiciones de trabajo en salud", en las bases de datos Medline, Lilacs y SciELO. Se incluyeron 19 artículos de 1999 a 2009, que abordaron temas sobre los impactos de los cambios en el proceso de trabajo y en la salud de los trabajadores de la atención a la salud. Los resultados indicaron que existe un proceso de enfermedad de los profesionales de la salud, debido a las consecuencias provocadas por la reestructuración. Se han identificado como causas: hora doble de trabajo, bajos salarios, alta carga de trabajo, falta de autonomía, subordinación al modelo médico-hegemónico actual y presiones de los jefes y de la familia de los usuarios, lo que refleja en la calidad de vida del profesional y en la asistencia. Se sugiere un mayor diálogo y apoyo de los gerentes, para que sean conocidas las lagunas y sugerencias sobre el proceso de trabajo, y la inversión en las relaciones interpersonales en el equipo multidisciplinario, por lo que cada uno tiene una mayor autonomía en el trabajo. También es necesario invertir en el espacio relacional trabajador-usuario, con acciones de acogida y humanización de la atención.

Palabras clave: Reestructuración productiva; Proceso de trabajo en salud; Proceso salud-enfermedad en salud

\section{INTRODUÇÃO}

\section{1 Relação Saúde - Trabalho}

O trabalho é um processo no qual o homem transforma os recursos da natureza através da sua atividade, conferindo-lhes utilidade às necessidades humanas e, também transforma a si e às suas relações interpessoais, através do contato com outras pessoas. ${ }^{(1)} \mathrm{O}$ processo de trabalho compreende a força de trabalho, o objeto a ser transformado e os meios utilizados para tal fim. Ele engloba condições objetivas (objetos e meios) e subjetivas, que estão presentes através das expectativas e significados que o ser humano dá ao trabalho exercido. ${ }^{(1,}$

2) Neste contexto, a organização do trabalho compreende três elementos: o conteúdo do trabalho, a forma como é feito e as relações interpessoais. As diferentes interações entre esses três elementos determinaram os processos produtivos existentes. ${ }^{(1,2)}$

\subsection{Processo de Trabalho no Setor Saúde}

As mudanças nos processos produtivos abordados anteriormente tiveram início e foram mais nítidas nas indústrias, principalmente automotiva. Entretanto, elas trouxeram 
consigo mudanças sociais e econômicas que afetaram também outros setores. O setor saúde é basicamente trabalho do setor de serviços, inserido no setor terciário da economia. Como tal, ele tem seu processo de trabalho constantemente modificado, de acordo com as mudanças sociais e econômicas vivenciadas pela sociedade. Porém, uma característica permanece: a produção e o consumo são indissociáveis, pois acontecem simultaneamente. De acordo com Pires: "o produto final é a própria prestação da assistência de saúde que é produzida no momento que é consumida"(5)

O modelo atual de gestão em saúde é centrado no médico, denominado "modelo médico-hegemônico". O que prevalece atualmente no processo de trabalho do médico é a sua concentração nas tecnologias duras (representadas pelos procedimentos e instrumentos tecnológicos existentes) e leve-duras (que são os saberes e conhecimentos adquiridos da sua área de atuação), em detrimento das tecnologias leves, representadas pelo ponto de interseção entre médico e usuário e suas relações sociais. ${ }^{(6)}$

Neste modelo hegemônico, o foco principal, a partir das necessidades reais do usuário, é colocado no interesse do capital, representado pelas indústrias farmacêutica, tecnológica (responsáveis pela tecnologia encontrada nos inúmeros exames complementares existentes) e pelas seguradoras de saúde, que movimentam a maior parte dos recursos que o setor saúde gera. O trabalho se concentra nas tecnologias duras e leve-duras.

As características da flexibilização do trabalho estão presentes no setor saúde, a exemplo da terceirização e do trabalho sem carteira assinada. ${ }^{(5)}$ Do outro lado, o trabalho subordinado ao modelo hegemônico, parcelado, precarizado, não dá qualidade de vida ao trabalhador, e pelo contrário, o deixa sujeito a plantões exaustivos, necessidade de dois ou mais empregos para garantir o seu sustento, o desemprego e alta demanda de trabalho para poucos funcionários.

Nesta perspectiva este estudo tem como objetivo pesquisar as publicações acerca das mudanças no processo de trabalho do setor saúde a fim de identificar os impactos trazidos aos profissionais de saúde e ao processo saúde-doença.

\section{METODOLOGIA}

Foi realizada uma revisão da literatura acerca do tema "reestruturação produtiva e o trabalho em saúde", publicadas entre 1999 e 2009, nas bases de dados Lilacs, Medline e 
Scielo. Foram utilizados os descritores "processo de trabalho em saúde", "reestruturação produtiva", "reestruturação hospitalar" e "condições de trabalho em saúde".

Os critérios de inclusão foram: publicações feitas entre 1999 e 2009 e artigos cujo tema abordasse o processo de trabalho e o processo saúde-doença do profissional de saúde, especificamente. Não houve direcionamento a uma única categoria profissional, portanto, foram incluídos todos os profissionais que integram a equipe de saúde, inclusive os agentes comunitários. Também não foi especificada a complexidade do atendimento, podendo abordar tanto a atenção básica e o atendimento domiciliar, quanto o atendimento hospitalar.

O critério de exclusão englobou os temas que abordassem categorias profissionais que não fossem envolvidos com a atenção direta à saúde, (como, por exemplo, os auxiliares administrativos). Foram excluídos os artigos que abordassem apenas os custos financeiros da reestruturação produtiva nos hospitais, não abordando aspectos como qualidade e condições de trabalho dos profissionais da atenção à saúde.

Os artigos selecionados foram analisados de acordo com as seguintes variáveis: periódico e país de publicação, ano da publicação, qualificação profissional dos autores, profissões analisadas, temas abordados, enfoque metodológico, sugestões das pesquisas e o impacto provocado pela reestruturação produtiva no processo saúde-doença e no processo de trabalho dos profissionais de saúde.

\section{RESULTADOS}

Foram selecionados quarenta artigos, após a leitura do título e resumo. Destes, vinte e um foram excluídos após a leitura completa, por estarem dentro dos critérios de exclusão. Foram selecionados dezenove artigos, sendo doze nacionais e sete internacionais. Destes últimos, quatro foram oriundos do Canadá. As demais foram da Austrália, Nova Zelândia e Suécia.

Os periódicos das publicações dispostos na Tabela 1 que mais abordaram o tema foram: Journal of Nursing Management e The Health Care Manager, no âmbito internacional, e nas revistas nacionais foram a Ciência e Saúde Coletiva, seguida de Caderno de Saúde Pública, Revista Brasileira de Enfermagem e Revista da Escola de Enfermagem da USP. 
Tabela 1 - Frequência de publicações segundo periódicos indexados nas bases de dados do Scielo, Medline e Lilacs, período 1999-2009.

\begin{tabular}{lcc}
\hline Periódicos & Número de artigos (N=19) & $(\boldsymbol{\%})$ \\
\hline Ciência e Saúde Coletiva & 03 & 16,3 \\
Caderno de Saúde Pública & 02 & 10,5 \\
Journal of Nursing Management & 02 & 10,5 \\
Revista Brasileira de Enfermagem & 02 & 10,5 \\
Revista de Escola de Enfermagem da USP & 02 & 10,5 \\
The Health Care Manager & 02 & 10,5 \\
Acta Paulista de Enfermagem & 01 & 5,2 \\
Australian Journal of Advanced Nursing & 01 & 5,2 \\
International Journal of Health Services & 01 & 5,2 \\
Medical Care & 01 & 5,2 \\
Revista de Saúde Coletiva & 01 & 5,2 \\
Revista latino-americana de enfermagem & 01 & 5,2 \\
\hline Fonte: Base de dados do Scielo, Medline e Lilacs
\end{tabular}

Observa-se na Tabela 2, o número de artigos por ano de publicação. Pode-se notar que houve um aumento das pesquisas, evidenciando uma crescente preocupação com o tema entre os profissionais de saúde e pesquisadores. Isto pode ser creditado à falta de informação acerca do tema na área de saúde, que prioriza as ações de assistência e de reabilitação em detrimento das ações prevenção.

Tabela 2 - Frequência de publicações indexadas nas bases de dados do Scielo, Medline e Lilacs segundo ano de publicação, período 1999-2009.

\begin{tabular}{lcc}
\hline Ano de publicação & Número de Artigos $(\mathbf{N}=\mathbf{1 9})$ & $\mathbf{( \% )}$ \\
\hline 1999 & 01 & 5,3 \\
2000 & 01 & 5,3 \\
2001 & 01 & 5,3 \\
2002 & 01 & 5,3 \\
2003 & 01 & 5,3 \\
\hline
\end{tabular}




\begin{tabular}{lcc}
\hline 2004 & - & - \\
2005 & 03 & 15,8 \\
2006 & 03 & 15,8 \\
2007 & 03 & 15,8 \\
2008 & 01 & 5,3 \\
2009 & 04 & 20,8 \\
\hline
\end{tabular}

Sobre as profissões abordadas em cada artigo, a equipe de enfermagem foi a mais pesquisada entre os artigos selecionados. Este fato pode ser conseqüência de duas variantes: por um lado, há um número crescente de enfermeiros pesquisadores; por outro, ela é uma equipe multiforme, que envolve tanto enfermeiros quanto técnicos e auxiliares de enfermagem, conseqüentemente, responsável por uma grande parte dos cuidados em saúde e por isso mais exposta a riscos ocupacionais e à precarização do trabalho (Tabela 3). Também foram estudados os profissionais que atuam na atenção domiciliar, agentes comunitários e profissionais atuantes na atenção básica. Dois artigos abordaram o tema de forma ampla, focando no profissional de saúde em geral.

\section{Tabela 3 - Frequência de profissões estudadas nas publicações indexadas nas} bases de dados do Scielo, Medline e Lilacs, período 1999-2009.

\begin{tabular}{lll}
\hline Áreas profissionais pesquisadas & $\mathbf{N}$ & $(\boldsymbol{\%})$ \\
\hline Enfermagem & 12 & 63,1 \\
Profissionais atuantes na Assistência & 03 & 15,8 \\
domiciliar & 02 & 10,5 \\
Profissionais de Saúde & 01 & 5,3 \\
Agente comunitário & 01 & 5,3 \\
Profissionais atuantes na Unidade &
\end{tabular}

O enfoque metodológico de cada pesquisa está representado na Tabela 4. Houve um equilíbrio entre as pesquisas qualitativas e quantitativas, ambas com 36,8\%. Seguiram-se as revisões bibliográficas $(15,7 \%)$ e os estudos de caso $(10,7 \%)$.

\section{Tabela 4 - Enfoque Metodológico das publicações indexadas nas bases de dados do Scielo, Medline e Lilacs, período 1999-2009.}




\begin{tabular}{lll}
\hline Metodologia & N & $(\boldsymbol{\%})$ \\
\hline Pesquisa Qualitativa & 07 & 36,8 \\
Pesquisa Quantitativa & 07 & 36,8 \\
Revisão bibliográfica & 03 & 15,7 \\
Estudo de caso & 02 & 10,7 \\
\hline Fonte: Base de dados do Scielo, Medline e Lilacs & &
\end{tabular}

Evidencia-se na Tabela 5, o impacto provocado pelas mudanças no processo produtivo no setor saúde. Cinco artigos identificaram através de seus resultados um aumento do estresse e da insatisfação no trabalhado entre os profissionais de saúde. Outros seis chegaram à conclusão de que houve mudanças estruturais no processo de trabalho após a reestruturação, afetando, inclusive, a qualidade dos serviços prestados aos usuários. Estes foram os principais resultados obtidos. Outros resultados foram observados, incluindo aumento das demissões, aumento da incidência de doenças crônicas devido aos fatores estressantes e às condições do trabalho, como Diabetes Mellitus (DM), Hipertensão Arterial Sistêmica (HAS) e Dislipidemias, aumento da divisão social entre o trabalho manual e intelectual, precarização e terceirização e aumento dos sentimentos de medo, raiva, preocupação e revolta diante da exposição ocupacional.

\section{Tabela 5 - Impacto Provocado pela Reestruturação Produtiva no Setor Saúde, segundo os resultados das publicações indexadas nas bases de dados do Scielo Medline e Lilacs, período 1999-2009}

\begin{tabular}{lcc}
\hline Impactos & N=19 & (\%) \\
\hline $\begin{array}{l}\text { Mudanças estruturais no processo de trabalho, afetando o atendimento aos } \\
\text { usuários. }\end{array}$ & 06 & 31,5 \\
Aumento do stress e insatisfação no trabalho & 05 & 26,3 \\
Aumento da divisão social do trabalho & 03 & 15,8 \\
Aumento das demissões & 02 & 10,5 \\
$\begin{array}{l}\text { Aumento da incidência de doenças crônicas entre os trabalhadores (DM, HAS, } \\
\text { dislipidemia) }\end{array}$ & 01 & 5,3 \\
$\begin{array}{l}\text { Precarização e terceirização } \\
\text { Sentimentos de medo, raiva, preocupação e revolta diante da exposição }\end{array}$ & 01 & 5,3 \\
ocupacional. & 01 & 5,3 \\
\hline Fonte: Base de dados do Scielo, Medline e Lilacs &
\end{tabular}

Os artigos sugerem algumas mudanças no atual sistema de trabalho no setor saúde (Tabela 6). Sete artigos indicaram que um maior apoio da chefia, através da escuta das 
opiniões dos funcionários, seria um benefício que traria confiança e respeito dos trabalhadores, como aumento da sensação de valorização pelos mesmos.

Tabela 6 - Sugestões oferecidas pelos autores dos artigos, de acordo com seus achados. Publicações indexadas nas bases de dados do Scielo, Medline e Lilacs, período 1999-2009

\begin{tabular}{lcc}
\hline Sugestões & $\mathbf{N = 1 9}(\boldsymbol{\%})$ \\
\hline Melhorar o apoio da chefia com melhora da comunicação com os funcionários & 07 & 36,8 \\
Assegurar melhores condições e relações de trabalho e estabilidade & 03 & 15,8 \\
Mudanças no espaço físico, na carga de trabalho e nos recursos humanos & 02 & 10,5 \\
Priorizar as relações das tecnologias leves e o trabalho em equipe & 02 & 10,5 \\
$\begin{array}{l}\text { Valorizar as opiniões e o saber técnico dos ACS e demais membros das equipes } \\
\text { multidisciplinares }\end{array}$ & 02 & 10,5 \\
Aumentar a autonomia da equipe multidisciplinar no tratamento do usuário & 01 & 5,3 \\
Incentivar a normatização da acreditação hospitalar & 01 & 5,3 \\
Maiores reflexões acerca do tema proposto e maior engajamento sindical & 01 & 5,3 \\
\hline \multicolumn{1}{l}{ Fonte: Base de dados do Scielo, Medline e Lilacs }
\end{tabular}

Fonte: Base de dados do Scielo, Medline e Lilacs

Melhoria das condições e relações de trabalho, com geração de estabilidade no emprego, também foi um ponto importante discutido entre os autores. Essas condições de trabalho estão diretamente envolvidas com mudanças no espaço físico, e no aumento de recursos humanos para diminuição da carga de trabalho. Outras sugestões dadas foram: priorizar as tecnologias leves e o trabalho em equipe, aumentar as reflexões acerca do tema e o engajamento sindical na luta pelos direitos trabalhistas, incentivar a normatização da acreditação hospitalar, aumentar a autonomia da equipe multidisciplinar no atendimento ao usuário e, em relação ao trabalho do agente comunitário de saúde, a proposta foi a de valorizar as opiniões e o saber técnico do mesmo, inserindo-o na tomada de decisões acerca dos tratamentos aos usuários.

\section{DISCUSSÃO}


Os artigos abordaram temas variados, porém todos com vistas à análise dos processos de trabalho dos diversos profissionais de saúde diante da reestruturação produtiva.

\subsection{Organização do trabalho}

O estudo realizado por Pires ${ }^{(5)}$ menciona os principais fatores e conseqüências da reestruturação para a categoria de saúde. Destaca a introdução de novas tecnologias; a redução da força de trabalho qualificada; a exigência de aumento do rendimento; a redução dos salários; a terceirização e a fragmentação do trabalho, com pouco incentivo à interdisciplinaridade. Enfatiza o uso da tecnologia como principal parâmetro da reestruturação produtiva, e indica, como vantagem, a maior rapidez, segurança e confiabilidade no diagnóstico e terapêutica. Em contrapartida, a desvantagem é que foi criado um abismo entre o saber técnico e o saber empírico do usuário, que não tem condições de avaliar as decisões médicas. Assim, o uso de tecnologias leves, o espaço relacional entre trabalhador-usuário, ficou em segundo plano, com a supervalorização das tecnologias duras.

Outros artigos corroboram com os achados de Pires. O estudo realizado por Duffield et $\mathrm{al}^{(7)}$ analisa o impacto da reestruturação no setor de enfermagem. Ele faz uma revisão de literatura, onde explica que a reestruturação ocorrida na Austrália trouxe um movimento de fusões e diminuição de custos, a fim de aumentar a eficiência e lucratividade, fato ocorrido também nos demais países estudados (Canadá, Nova Zelândia e Suécia). Tornou-se comum a diminuição de leitos, de permanência no hospital e de integrantes da equipe, aumentando a carga de trabalho dos enfermeiros que permaneceram. O resultado foi uma insatisfação dos coordenadores de enfermagem - que ficaram presos a atos administrativos - e dos enfermeiros assistenciais, que tiveram a carga e o volume de trabalhos aumentados, com diminuição da autonomia, o que gerou descontentamento, a ponto de aumentar o número de demissões voluntárias.

\subsection{Satisfação}

Duffield et $\mathrm{al}^{(7)}$ afirmam que melhorar a satisfação dos profissionais ao garantir o acesso deles ao empoderamento e apoio organizacional deve se constituir prioridade para as chefias hospitalares. Sugerem que, para isso, sejam encontradas oportunidades para os 
profissionais contribuírem para a tomada de decisões estratégicas e autonomia para suas ações, através da valorização e disseminação de suas idéias.

\subsection{Adoecimento}

O adoecimento também foi objeto de pesquisas. Estudos realizados no Canadá retratam a realidade dos enfermeiros após a reestruturação produtiva e ressaltam as conseqüências psicológicas para os mesmos. O adoecimento é tema do estudo de Burke, ${ }^{(9)}$ uma pesquisa quantitativa que avalia a incidência da chamada "doença do sobrevivente", caracterizada por sintomas comportamentais e que incluem: baixo ânimo, pouco comprometimento com o trabalho, maior ceticismo, desconfiança e raiva. $\mathrm{O}$ autor concluiu que a sobrecarga de trabalho está diretamente relacionada à insatisfação e ao aparecimento destes sintomas e de outras doenças, como a depressão. Outra doença, avaliada no estudo de Greenglass e Burke, ${ }^{(10)}$ é a chamada "síndrome do burnout", ou a completa exaustão física e psicológica proveniente do trabalho. A exaustão psicológica é constituída de três aspectos: a exaustão emocional, o sentimento de despersonalização e a falta de realização pessoal.

$\mathrm{O}$ aumento dos casos de doenças ocupacionais e acidentes do trabalhos é fato relevante. O processo de trabalho atual é caracterizado por ritmo e jornada acelerados, recursos materiais inadequados, falta ou insuficiência de equipamentos de proteção individual (EPI), falta de interesse do empregador em investir em equipamento de proteção coletiva, escassez de profissionais com grande volume de tarefas e poucas pausas nas jornadas, dentre outros. Este quadro, aliado aos fatores desencadeantes de estresse ocupacional, coloca o profissional em risco de acidentes com material biológico e com material perfuro-cortante, além do risco de desenvolver doenças crônicas.

A pesquisa de Sarquis e Felli ${ }^{(11)}$ avalia as percepções dos trabalhadores acerca dos sentimentos vivenciados após um acidente ocupacional. O primeiro sentimento relatado foi o medo, expresso de três formas; medo de perder o emprego, medo da reação da chefia e medo de adoecer. Devido ao primeiro, muitos profissionais deixam de notificar o acidente. Muitos deles, temendo a não aceitação, escondem o ocorrido dos seus familiares, prejudicando sua qualidade de vida e vivência pessoal. Os outros sentimentos são a raiva, a revolta e a culpa, pois muitos acham que o acidente ocorreu devido ao seu próprio descuido, perpetuando a concepção do ato inseguro e jogando a culpa para o trabalhador. $\mathrm{O}$ artigo conclui que o 
acidente não atinge apenas o profissional, mas também os familiares, colegas e chefes de trabalho e sugere que haja mudanças estruturais nos processos de trabalho.

O estudo de Teixeira e Mantovani ${ }^{(12)}$ avaliou os fatores de risco para doenças crônicas presentes na organização do trabalho em saúde e as medidas de prevenção. Entre os fatores de risco identificados estão a diminuição do número de profissionais, excesso de atividades, estresse, falta de alimentação adequada, predispondo à obesidade, risco de acidentes, longas jornada, baixa remuneração, duplo ou triplo empregos, dentre outros. Verificou-se um aumento de doenças crônicas como HAS, diabetes mellitus e dislipidemias entre os profissionais de enfermagem. Como medidas de atenuação, observou-se que alguns desses profissionais, após o diagnóstico dessas doenças, optaram por melhorar a qualidade de vida, através medidas pessoais (diminuição de peso, realização de atividades físicas e adesão ao tratamento medicamentoso) e profissionais, como a renúncia a metas profissionais, diminuição de envolvimento com o paciente, diminuição da carga horária e da carga de trabalho, inclusive com pedidos de demissão.

\subsection{Gestão do trabalho e cuidado em saúde}

A contratação de auxiliares de enfermagem em maior número para substituir enfermeiros, como forma de redução de custos, foi apontada como um fator estressante adicional pelo estudo de Greenglass e Burke, pois, não só os enfermeiros se vêem ameaçados a perder o emprego, como também tem que treinar e supervisionar o trabalho das auxiliares. Outro fator estressante apontado na pesquisa está na sensação de traição sentida pelos funcionários mais compromissados com o emprego, que sentem que não conseguiram retribuição justa pelo trabalho em prol da instituição, ao contrário, alguns receberam a demissão em troca. A pesquisa concluiu que a reestruturação produtiva realmente contribuiu para a exaustão dos enfermeiros, sendo a exaustão emocional a mais prevalente. ${ }^{(10)}$

Sobre os motivos de demissão na área de saúde, dois artigos associaram estes motivos com as mudanças ocorridas com a reestruturação produtiva. Estudo de Anselmi et al ${ }^{(13)}$ realizado no Brasil, abordou o tema rotatividade no emprego de enfermagem. Os resultados mostraram uma alta rotatividade nos postos de enfermeiro e auxiliar de enfermagem, nos quais, respectivamente, $27,8 \%$ e $23,5 \%$ mantiveram seus empregos após cinco anos no setor. Em contraste, o emprego de técnico de enfermagem foi o que apresentou maior estabilidade, com $89,5 \%$ de permanência no emprego após o mesmo período. As causas que os autores 
apontaram para tal resultado estão na ampliação dos postos de trabalho na Atenção Básica e Programas de Saúde da Família, que oferecem horários mais flexíveis e melhores condições de trabalho para os enfermeiros e auxiliares, o que pode ter causado migração destes profissionais para as Unidades Básicas. Além disso, a precarização do trabalho não constitui atrativo para a permanência no serviço hospitalar. A pesquisa conclui que, para diminuir esta rotatividade, é preciso assegurar a estabilidade do emprego, com melhores condições de trabalho para estes profissionais.

$\mathrm{O}$ estudo de Skytt et $\mathrm{al}^{(\mathbf{1 4})}$ corrobora com o primeiro, por creditar à precarização do trabalho o motivo de pedidos de demissão voluntária de enfermeiros da atenção hospitalar. Os autores apresentaram algumas sugestões trazidas pelos entrevistados, como: maior apoio da direção, necessidade de melhora dos sistemas computadorizados, oportunidades de crescimento e educação continuada, comunicação, apoio e opinião efetivos dos superiores, criação de uma atmosfera de confiança, valor e auto-estima com a equipe. Eles concluíram que as mudanças organizacionais foram o principal motivo apontado para as demissões voluntárias e a falta de apoio e a relação com os superiores foi um fator que influenciou na decisão.

A equipe de Saúde da Família (PSF) é responsável por acompanhar o processo saúdedoença da população abrangida pelo seu setor e propor medidas de prevenção e controle dos casos. Encontra-se a figura do agente comunitário de saúde (ACS), que se constitui um trabalhador de nível médio ou técnico, responsável por ir ao domicílio dos usuários para verificar o estado geral através da medição dos sinais vitais e promover medidas de prevenção em usuários identificados como grupo de risco. ${ }^{(15)}$ Neste sentido, foi realizado um estudo ${ }^{(15)}$ de caso, onde foi acompanhado o cotidiano de uma equipe de PSF e especificamente, dos ACS.

A conclusão foi que a opinião e a participação dos ACS na organização dos serviços não são valorizadas pela equipe, que tenta desqualificar sua atuação, procurando detê-los nos procedimentos normativos do modelo hegemônico, por acharem que eles não são capazes de realizar nada além do que for mandado. Com isso, a equipe aumenta o abismo entre a divisão técnica e social do trabalho manual e o intelectual. Os ACS se sentem desvalorizados em relação à equipe. Eles procuram validar seu trabalho criativo através de ações de promoção e educação em saúde, na tentativa de burlar as normas nas quais são pautadas o seu processo de trabalho. ${ }^{(15)}$ 
No Brasil, a atenção domiciliar é considerada por alguns autores como dispositivo da reestruturação produtiva, no sentido de produzir novas formas de cuidado aos usuários e nova organização de trabalho. O estudo de Skytt et al, ${ }^{(6)}$ teve como objetivo analisar os processos e tecnologias do trabalho no Programa de Atenção Domiciliar (PAD) e suas contribuições para mudanças no cenário atual da saúde. Abordou vários aspectos peculiares do PAD e como eles afetam o processo de trabalho neste setor.

Em relação à gestão do trabalho, verificou-se que a empresa gestora do PAD possui empregados contratados e terceirizados. Eles recebem incentivos através de premiação dos trabalhadores mais engajados e o estímulo ao estudo superior para técnicos de enfermagem com mais de três anos na casa. Há também o treinamento continuado acerca de relações interpessoais, comportamento e ética.

Em relação à micropolítica do processo de trabalho, a figura central é a do médico, que tem a função assistencial aliada à administrativa, uma vez que é ele quem faz o primeiro contato com o usuário e indica que cuidados serão necessários, coordenando, assim, o trabalho dos outros profissionais como enfermeiros, fisioterapeutas, nutricionistas, assistentes sociais, etc. Entretanto, uma vez estabelecido o tratamento, o enfermeiro passa a ser o centro do processo de trabalho, já que ele se torna o responsável pela intermediação com os demais profissionais, inclusive com a tarefa de relatar o progresso ao médico, e é também o principal vínculo da operadora com o usuário.

$\mathrm{O}$ estudo de Backes et $\mathrm{al}^{(22)}$ traz o tema da humanização centrada no trabalhador. Foi realizada uma pesquisa para avaliar a implantação de um processo de humanização em um hospital, através de entrevistas com funcionários. Foi concluído que um indicativo de humanização no ambiente de trabalho é a integração do trabalho interdisciplinar. Atitudes gentis, confraternizações e outras ações de acolhimento reforçam o sentimento de trabalho em equipe e são eficazes para maior valorização do trabalhador, o que melhora também o seu desempenho no trabalho ${ }^{(22)}$

\section{CONSIDERAÇÕES FINAIS}

A reestruturação produtiva trouxe mudanças significativas ao processo de trabalho dos profissionais de saúde. Como explica Merhy, ${ }^{(6)}$ vive-se no tempo em que as valises das tecnologias duras e leve-duras se sobrepõem e subjugam a valise das tecnologias leves, o 
espaço relacional entre trabalhador e usuário. Dá-se mais importância aos laudos dos exames complementares do que à escuta das queixas principais trazidas pelos usuários.

As mudanças nos processos de trabalho dos profissionais de saúde trouxeram algumas conseqüências negativas à saúde dos mesmos, provocando adoecimento físico e emocional. A precarização das condições de trabalho dos enfermeiros chega ao ponto em que coloca em risco o bem-estar do usuário, pois, com a baixa qualificação da equipe, a sobrecarga de trabalho dos enfermeiros aumenta significativamente, e com ela os níveis de estresse físico e emocional e a insegurança no trabalho.

É possível perceber que o modelo médico-hegemônico ainda prevalece na assistência à saúde. A falta de diálogo, a superioridade entre os membros da equipe multidisciplinar, e a subestimação do conhecimento e das opiniões dos diversos profissionais de saúde causam uma ruptura nas relações interpessoais e refletem na qualidade do atendimento.

As pesquisas apontaram que a melhor forma de garantir melhores condições de trabalho e aumento da satisfação no emprego é o diálogo. Este deve começar entre os profissionais e os gestores, para que ambos cheguem a conclusões acerca de melhorias para o atendimento e para a realização do trabalho em si. Ele também se mostra muito importante para o estabelecimento de relações interpessoais entre a equipe multidisciplinar, para encorajar o respeito e valorização das idéias. Por fim, o diálogo entre o trabalhador com o usuário é de extrema importância para ambos, pois, o usuário irá se sentir acolhido, escutado e verá que suas necessidades serão atendidas e o profissional irá ter condições de atuar de forma satisfatória.

Ressalta-se a importância de novos estudos sobre as causas e conseqüências da precarização do trabalho no setor saúde, sendo de extrema importância o incentivo à pesquisa, a procura de soluções e a educação continuada dos profissionais e estudantes deste campo.

\section{REFERÊNCIAS}

1. Aguiar ZN. Processo de trabalho e algumas implicações para a saúde do trabalhador. In: RIBEIRO Maria C.S: A enfermagem e o trabalho: fundamentos para a atenção à saúde dos trabalhadores. São Paulo: Martinari, 2008. p. 13-17.

2. Ribeiro CVS, Léda DB. O significado do trabalho em tempos de reestruturação produtiva. Estud. pesqui. psicol. 2004;4(2):76-83.

3. Silva AC. Aspectos psicossociais do trabalho e transtornos mentais comuns entre trabalhadores informais. Dissertação de mestrado. Bahia: Departamento de Saúde. Programa 
de Pós-Graduação em Saúde Coletiva. Universidade Estadual de Feira de Santana, UEFS, 2007.

4. Merlo ÁRC, Lapis NL. A saúde e os processos de trabalho no capitalismo: reflexões na interface da psicodinâmica do trabalho e da sociologia do trabalho. Psicol. soc. 2007;19(1):61-8.

5. Pires D. Reestruturaçäo produtiva e conseqüências para o trabalho em saúde. Rev. bras. enferm. 2000;53(2):51-63.

6. Merhy EE. Um Ensaio sobre o Médico e suas Valises Tecnológicas: Fazendo um Exercício Sobre a Reestruturação Produtiva na Produção do Cuidado. In: MERHY Emerson. Saúde: a Cartografia do Trabalho Vivo. São Paulo: Hucitec, 2007. p 93-112.

7. Duffield $\mathrm{C}$ et al. The impact of hospital structure and restructuring on the nursing workforce. Aust. j. adv. nurs. 2007;24(4):42-6.

8. Mccloskey BA, Diers DK. Effects of New Zealand's health reengineering on nursing and patient outcomes. Medical Care. 2005;43(11):1140-6.

9. Burke RJ. Hospital restructuring stressors, support, and nursing staff perceptions of unit functioning. The Health Care Manager, 2005;24(1):21-28.

10. Greenglass ER, Burke RJ. Hospital restructuring and burnout. J Health Hum Serv Adm. 2002;25(1):89-114.

11. Sarquis LMM, Felli VEA. Os sentimentos vivenciados após exposição ocupacional entre trabalhadores de saúde: fulcro para repensar o trabalho em instituições de saúde. Rev. bras. enferm. 2009;62(5):701-42.

12. Teixeira RC, Mantovani MF. Enfermeiros com doença crônica: as relações com o adoecimento, a prevenção e o processo de trabalho. Rev. Esc. Enferm. USP. 2009;43(2):41521.

13. Anselmi ML, Duarte GG, Angerami ELS. "Sobrevivência" no emprego dos trabalhadores de enfermagem em uma instituição hospitalar pública. Rev. latinoam. enferm. 2001;9(4):13-8.

14. Skytt B, Ljunggren B, Carlsson M. Reasons to leave: the motives of first-line nurse managers' for leaving their posts. J. nurs. manag. 2007;15(3):294-302;

15. Ferreira VSC. et al. Processo de trabalho do agente comunitário de saúde e a reestruturação produtiva. Cad. saúde pública. 2009;25(4):898-906.

16. Bujdoso YLV et al. A academia e a divisão social do trabalho na enfermagem no setor público: aprofundamento ou superação? Ciênc. saúde coletiva. 2007;12(5):1363-74.

17. Campos CMS, Soares CB. A produção de serviços de saúde mental: a concepção dos trabalhadores. Ciênc. saúde coletiva. 2003;8(2):621-628. 
18. Denton Margaret et al. Job stress and job dissatisfaction of home care workers in the context of health care restructuring. Int. j. health serv. 2002;32(2):327-57.

19. Franco TB, MERHY Emerson E. Atenção domiciliar da saúde suplementar: dispositivo da reestruturação produtiva. Ciênc. saúde coletiva. 2008;13(5):1511-20.

20. Martins AA. et al. A produção do cuidado no programa de atenção domiciliar de uma cooperativa médica. Revista de Saúde Coletiva. 2009;19(2):457-74.

21. Lima SBS, Erdmann AL. A enfermagem no processo da acreditação hospitalar em um serviço de urgência e emergência. Acta paul. enferm. 2006;19(3):271-8.

22. Backes DS, Lunardi Filho WD, Lunardi VL. O processo de humanização do ambiente hospitalar centrado no trabalhador. Rev. Esc. Enferm. USP. 2006;40(2):221-7.

23. Franco TB, Bueno WS, Merhy EE. O acolhimento e os processos de trabalho em saúde: o caso de Betim, Minas Gerais, Brasil. Cad. saúde pública. 1999;15(2):345-53. 